\title{
Structure and compressive behavior of Lightweight Clay Aggregate particle: experiments and numerical simulations
}

\author{
Shuangshuang Xia ${ }^{1}$, Zhifu Shen ${ }^{1 *}$, Hongmei $\mathrm{Gao}^{1}$, and Zhihua Wang ${ }^{1}$ \\ ${ }^{1}$ School of Transportation Engineering, Nanjing Tech University, Nanjing 210009, China
}

\begin{abstract}
Lightweight Clay Aggregate (LWCA) is a kind of highly porous granular particle which is often used as lightweight backfilling geomaterials. However, the easy breakage of LWCA particle plays a negative role in its geotechnical application. This study focuses on the internal structure and compressive behavior of single LWCA particle using experiments and numerical simulations. The uniaxial compressive strength and loading stiffness of the LWCA particle is attributed to its special porous internal structure. The results could enrich our understanding in particle crushing.
\end{abstract}

\section{Introduction}

Lightweight Clay Aggregate (LWCA) is a kind of loose and porous lightweight granular material, which is made of shale, clay or fly ash by sintering at high temperature or adding gelling agent. LWCA has the characteristics of loose internal structure. Due to its low density, it is an ideal backfilling geomaterials. Its benefits include reducing settlement of embankment, reducing lateral earth pressure applied on retaining wall and increasing stability of geotechnical structure.

However, the highly porous structure of the particle makes it easy to crush under load. Previous studies have shown that the particle crushing of soil is related to the internal and external factors such as particle strength, size, shape, contact coordination number, gradation, compactness and stress condition [1-9]. In terms of LWCA particle, Calderia \& Neves [10] used a $500 \mathrm{~mm}$ diameter consolidator to measure compression behavior of LWCA assembly with particle diameter between 10 and $20 \mathrm{~mm}$ and it is found that LWCA assembly presents high plasticity bahavior with an unloading modulus more than 10 times greater than the loading modulus. However, the microscopic process of LWCA particle crushing is still not clear. Specifically, the internal structure of LWCA particle is quite different from that of sand particles studied in previous researches. If such a difference would significantly change the particle crushing behavior deserves detailed study. Therefore, this study focuses on the internal structure and compressive behavior of single LWCA particle using experiments and numerical simulations.

\section{Structure and mechanical behavior of LWCA particles}

\subsection{Internal structures of LWCA particles}

LWCA particles used in this study were sintered at high temperature in rotary kiln. The combustion of organic matter in the raw material forces the particles to expand, forming a honeycomb internal pore structure. The outer surface melts and sinters to form a hard shell. This process leads to a loose and porous light particle with a strong shell. The LWCA particles tested in our experiments were fired with clay as raw material, and the shape is mostly spherical or ellipsoidal with relatively smooth surface, as shown in Fig. 1. The particle diameter range is between 2.0 and $5.5 \mathrm{~mm}$. The natural bulk density of LWCA assembly is $0.4 \mathrm{~g} / \mathrm{cm}^{3}$, indicating an ultra-light LWCA. The apparent density of LWCA particle is $0.73 \mathrm{~g} / \mathrm{cm}^{3}$. According to the specific gravity of solid LWCA material of 2.55 , the porosity of LWCA is 0.71 .

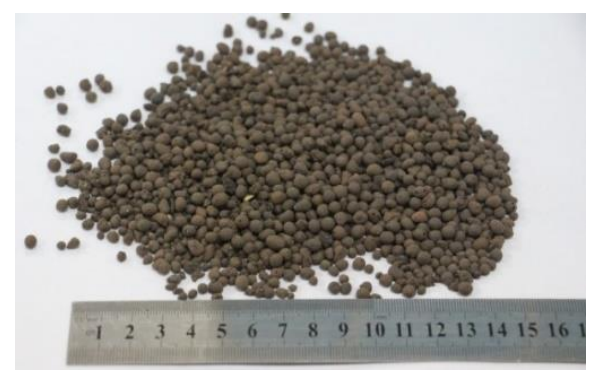

Fig. 1. LWCA particles used in the experiment.

Scanning Electron Microscope (SEM) and X-ray tomography images of LWCA particles were taken and shown in Fig. 2. From these images, the interior of LWCA particle is like a honeycomb structure. From the SEM image, the pores in the tested ultra-light LWCA are mostly spherical or ellipsoidal, and some of them are irregular. Most of the pores are isolated from each other

\footnotetext{
* Corresponding author: zhifu.shen@njtech.edu.cn

A video is available at https://doi.org/10.48448/k4he-5c20
} 
and only a small portion of the pores are connected with each other. There is a dense layer about $250 \mu \mathrm{m}$ thick on the particle surface and the pores in this layer are smaller than those inside. Thus, the porosity of the core is greater than that of the surface layer. Therefore, LWCA particle can be regarded as a kind of composite particle that are composed of a strong surface layer and a fragile core.

(a)

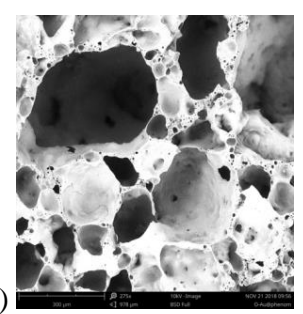

(b)

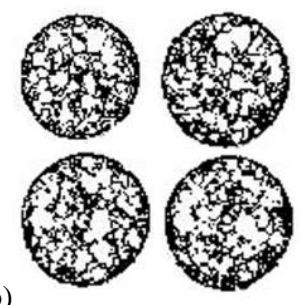

Fig. 2. Microscope images of LWCA particle: (a) SEM iamge, and (b) X-ray tomography image.

\subsection{Uniaxial compressive responses of LWCA particles in experiments}

A total of 300 LWCA particles were uniaxially compressed in an unconfined compression apparatus, which is a commonly available apparatus in geomechanics laboratory. These LWCA particles were tested individually in the apparatus. The apparatus was run electrically and the quasi-static loading rate is 0.025 $\mathrm{mm} / \mathrm{s}$. Typical compressive force - deformation curves are shown in Fig. 3. Generally, the pre-peak response is a linear curve but some deviations from a linear curve are also present, which are presumably attributed to the particle surface asperity. After the peak, a sharp decrease in the compressive force is observed.

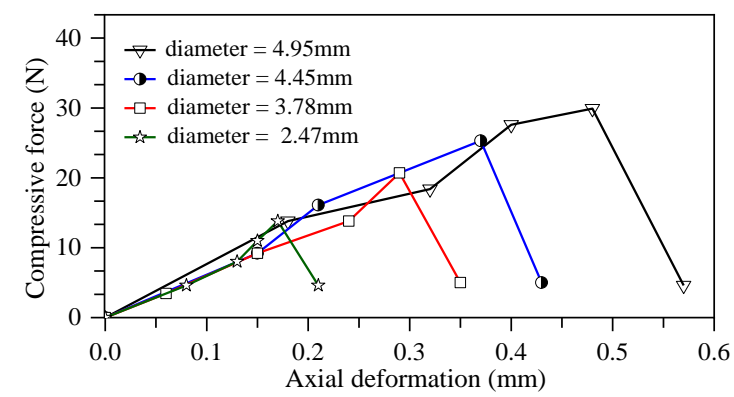

Fig. 3. Typical compressive force - axial deformation curves in experiments.

Fig. 4 and Fig. 5 summarize the peak compressive force and the loading stiffness in the experiments. The loading stiffness is defined as the peak compressive force divided by the axial deformation at the peak state. The discrete element modeling results are superimposed in the figures, which will be discussed later in this paper. Generally, the peak compressive strength increases nonlinearly with the LWCA particle size and the increasing rate becomes greater as the LWCA particle size increases. The loading stiffness generally decreases as the LWCA particle size increases. The possible reason is that LWCA is a kind of non-uniform particle with 'strong surface and weak core'. The smaller the particle is, the larger the specific surface area is, and the larger the volume proportion of the dense layer on the surface of LWCA, which helps the particles bear more external load. In addition, the pore size in the core of large particles is larger, which is negative to bear external load. Very large scattering in the experimental data was observed, which will be discussed later in this paper.

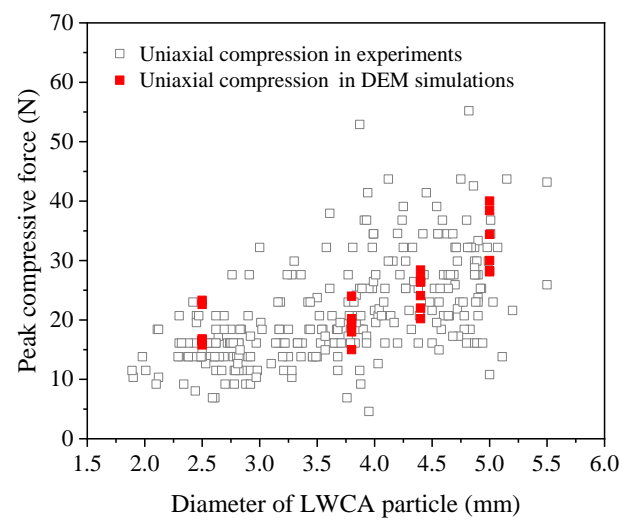

Fig. 4. Relationship between peak compressive force and LWCA particle size under uniaxial compression test.

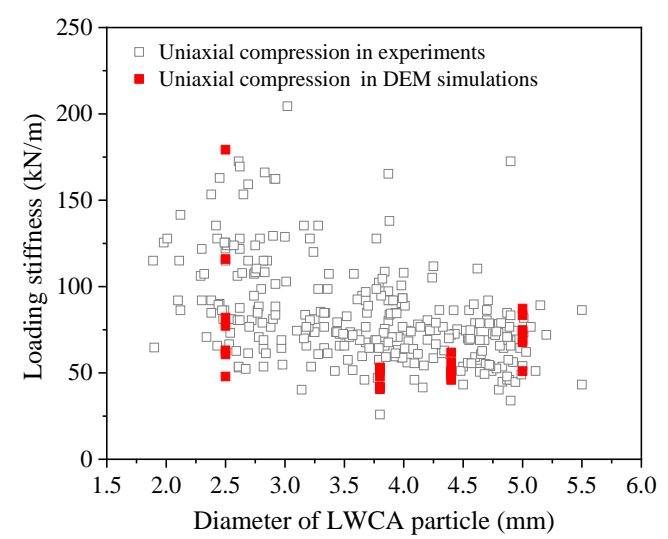

Fig. 5. Relationship between loading stiffness and LWCA particle size under uniaxial compression test.

The well-established Weibull distribution [5] is used to fit the experimental data:

$$
\ln \left[\ln \left(\frac{1}{P_{\mathrm{s}}}\right)\right]=m \ln \left(\frac{\sigma_{\mathrm{c}}}{\sigma_{0}}\right)
$$

where $P_{\mathrm{s}}$ is the survival probability of particles under a characteristic tensile stress of $\sigma_{\mathrm{c}}, m$ is the Weibull modulus, and $\sigma_{0}$ is the value of characteristic tensile stress when $37 \%$ of the tested LWCA particles survive.

The characteristic tensile stress is defined as

$$
\sigma_{\mathrm{c}}=0.9 \frac{F}{d^{2}}
$$

where $F$ is the compressive force and $d$ is the particle diameter.

Fig. 6 presents the normalized survival curves of particle size group 2.0-3.0 mm for example. The fitting parameters are: $\sigma_{0}=2.2 \mathrm{MPa}, m=3.80$ for group 2.0$3.0 \mathrm{~mm} ; \sigma_{0}=1.7 \mathrm{MPa}, m=3.17$ for group $3.0-3.5 \mathrm{~mm}$; $\sigma_{0}=1.5 \mathrm{MPa}, m=3.02$ for group 3.5-4.0 mm; $\sigma_{0}=1.3$ $\mathrm{MPa}, m=4.34$ for group $4.0-4.5 \mathrm{~mm} ; \sigma_{0}=1.1 \mathrm{MPa}, m$ $=4.62$ for group $4.5-5.0 \mathrm{~mm}$. Fig. 6 shows that the experimental data can be well fitted by the Weibull 
distribution. The parameter $\sigma_{0}$ decreases with the increase of particle size; the Weibull modulus $m$ generally increases with particle size, with an exception for the particle size group 2.0-3.0 $\mathrm{mm}$. The Weibull modulus falls in a range of 3.02 to 4.62 , close to the reported value of quartz sand.

The particle crushing modes are shown in Fig. 7. Local crushing at the loading point can be observed and the local crushing could be significant when the loading point is located at or near a large internal pore. Apart from the local crushing, an overall crushing can be widely observed. The whole LWCA particle can be fractured into two parts as shown in Fig. 7 (a), three parts as shown in Fig. 7 (b), or four parts as shown in Fig. 7 (c). Fig. 8 presents the percentages of the three crushing modes in the experiments and in the following numerical simulations. About $60 \%$ of the tested LWCA particles were crushed into two parts, and the other two modes have generally similar chance to be observed. The mechanism behind the observed crushing mode needs further investigation.

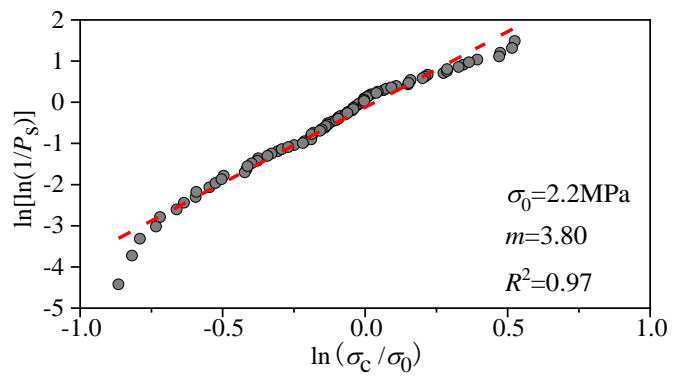

Fig. 6. Normalized survival curves with different particle sizes $(d=2.0-3.0 \mathrm{~mm})$.

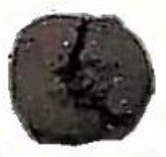

(a)

(b)

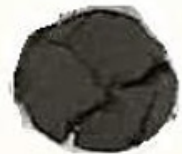

(c)

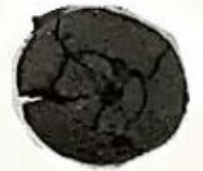

Fig. 7. Compressive crushing modes of LWCA particles in experiments: (a). two-part fracture, (b). three-part fracture, (c). four-part fracture.

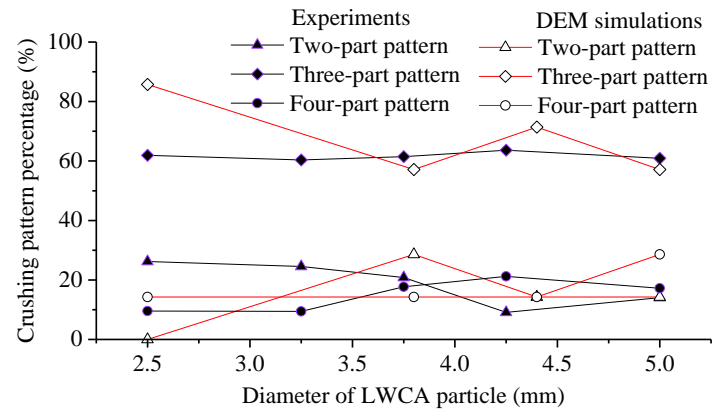

Fig. 8. Percentages of three crushing modes.

\section{Discrete element modelling of LWCA particles}

Discrete Element Method (DEM) simulation was used in this section to model the internal structure and crushing process of the LWCA particle under uniaxial compressive load. The strength of DEM simulation in capturing the discontinuous behavior of fragile material was utilized in this study.

\subsection{Discrete element modelling of LWCA particles}

The X-ray tomography images were binarized and a 3D linear interpolation function was used to determine if any spatial point corresponds to solid material or void. With this process, a 3D view of the LWCA particle can be prepared as shown in Fig. 9 (a). The following procedures were used to construct a particle-based discrete element model of a LWCA particle. Spheres were used as the basic particles.

(1) An assembly of basic particles were prepared to form a large sphere and equilibrium was reach. The basic particle's diameter was uniformly $0.04 \mathrm{~mm}$, and the spherical assembly has a diameter slightly larger than the counterpart real LWCA particle.

(2) The location of each basic particle is input into the 3D linear interpolation function. If the interpolated value is greater than 0.5 , the basic particle is regarded to represent solid and is kept; otherwise, the basic particle is deleted because it is regarded to be located at a void.

(3) After traversing all the basic particles and all the particles located at voids being deleted, the remaining particles are bonded by the parallel bond model in PFC3D[11] and the contact force was set to zero. These bonded particles constitute the numerical LWCA particle. The generated discrete element model of LWCA particle is shown in Fig. 9 (b). (a)

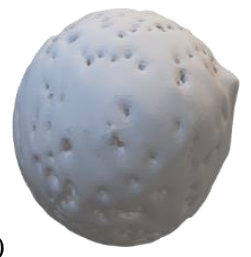

(b)

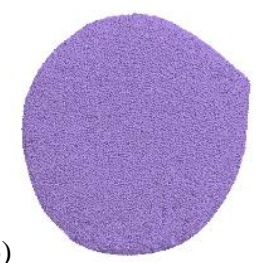

Fig. 9. Single particle model of LWCA: (a). X-ray tomography model; (b). DEM model.

The contact model parameter calibration/selection was performed as follows. The bond tensile strength and bond cohesion determines the peak compressive force of the numerical LWCA particle; they were simply assumed equal and were calibrated against the peak compressive force in experiment, leading to bond tensile strength $=$ bond cohesion $=60 \mathrm{MPa}$. The modulus of bond and modulus of contact control the contact stiffness between particles; there were assumed equal and were calibrated against the loading stiffness in experiment, leading to modulus of bond $=$ modulus of contact $=1 \mathrm{GPa}$. Note that a LWCA particle was represented by an assembly of spherical basic particles in our simulation, which could be over-discretized. To reach a compromise between the discrete and continuum features, the bond gap was set to be $10^{-3} \mathrm{~mm}$ so that a parallel bond contact can be created between two basic particles with a gap less than $10^{-3} \mathrm{~mm}$. In this way, more parallel contacts can be created and any single basic 
particle could be connected with surrounding basic particles through more parallel bond contacts. The coefficient of friction was chosen to be a common value of 0.5 without calibration. For details of the physical meanings of these parameters, please refer to [11].

\subsection{Simulated uniaxial compression response of LWCA particles}

The uniaxial compression test was simulated in DEM by enforcing two walls to move toward each other, as shown in Fig. 10. In this study, quasi-static compression was simulated with a very low compression rate of $4.1 \times 10^{-6} \mathrm{~mm} / \mathrm{s}$. The compressive force versus deformation were recorded during the simulation. When the vertical force dropped to $50 \%$ of the peak force, the simulation was terminated.

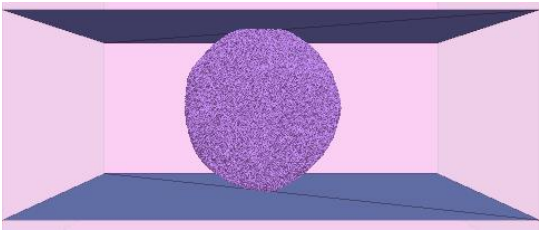

Fig. 10. LWCA single particle under uniaxial loading.

In this study, a total of four numerical LWCA particles were constructed from their respective X-ray tomography image sequences. The LWCA particle sizes are $2.5 \mathrm{~mm}, 3.8 \mathrm{~mm}, 4.4 \mathrm{~mm}$ and $5 \mathrm{~mm}$, respectively. Each LWCA particle was loaded uniaxially from seven directions. As a result, a total of 28 cases were simulated. Comparisons of peak compressive force and loading stiffness between the experiments and the numerical simulations are presented in Fig. 4 and Fig. 5. Generally, the numerical simulation can capture the variation of peak compressive force and loading stiffness with LWCA particle size. The variance of the numerical simulation for each particle size is smaller than the experiment results, which is presumably explained below. The data variance can be viewed as a combination of two sources: inter-particle variance and loading-direction variance. The inter-particle variance comes from the variation of internal honeycomb structure among LWCA particles; the loading-direction variance comes from the direction-dependent fracture resistance of LWCA particle due to the heterogeneity of the honeycomb structure within a single particle. Both the first and second sources of variance are present in the experiments while only the second source is present in the numerical simulations, which may explain the observed difference in data variance in the experiments and simulations.

Three types of particle crushing modes were observed in the numerical simulations, as shown in Fig. 11. In Fig.11, the basic particles that constitute the LWCA were colored by their respective velocity magnitude. In Fig. 11 (a), the LWCA particle was crushed into two parts and the fracture almost goes through the center of the particle. In Fig. 11 (b), the LWCA particle was crushed into three parts, and two parts are larger in size than the third one; the smallest fracture appeared the last. In Fig. 11 (c), a major fracture was first formed and it was followed by three minor fractures appearing almost simultaneously. There is no clear association between the fracture mode and the LWCA particle size or the compression loading direction. (a)

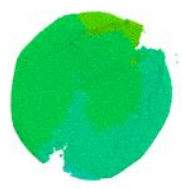

(b)

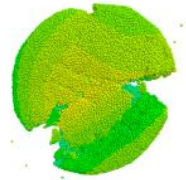

(c)

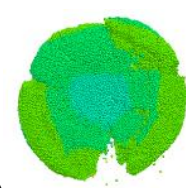

Fig. 11. Compressive crushing modes of LWCA particle in DEM simulations: (a). two-part fracture, (b). three-part fracture, (c). four-part fracture.

Fig. 8 shows that the percentages of the three crushing modes are similar in the experiments and numerical simulations. With the aid of DEM simulations in visualizing the crushing process and quantitatively accessing microscopic data, it would be the next step work to reveal the mechanism of the observed crushing mode.

The research is funded by Natural Science Foundation of the Jiangsu Higher Education Institutions of China with Grant No. 19KJB560015, National Natural Science Foundation of China for Young Researchers with Grant No. 51908284, and Natural Science Foundation of Jiangsu Province for Young Researchers with Grant No. BK20190667, which are sincerely appreciated. The authors would like to thank Prof. Mingjing Jiang in Tianjin University, China for his support in numerical simulations.

\section{References}

[1] Z. Y. Yin, Q. Xu, W. Hu, Chin. J. Geotech. Eng. 34, 2170-2180 (2012)

[2] J. R. Valdes, E. Koprulu, Géotechnique 58, 615622 (2008)

[3] M. R. Coop, K. K. Sorensen, T. B. Freitas, G. Georgoutsos, Géotechnique 54, 157-163 (2004)

[4] H. Shahnazari, R. Rezvani, Eng. Geol. 159, 98-105 (2013)

[5] G. R. McDowell, M. D. Bolton, Géotechnique 48, 667-679 (1998)

[6] Y. Nakata, Y. Kato, M. Hyodo, A. F. L. Hyde, H. Murata, Soils Found. 41, 39-51 (2001)

[7] K. Terzaghi, R. B. Peck, Soil mechanics in engineering practice, John Wiley and Sons Inc, 2, 65-67, New York, America (1948)

[8] A. J. Valsangkar, T. A. Holm, Geotech. Test. J. 22, 329-333 (1999)

[9] E. B. Hall, B. B. Gordon, Triaxial testing with large-scale high pressure equipment, Laboratory Shear Testing of Soils, ASTM (1964)

[10] L. M. M. S. Caldeira, E. M. D. Neves, J. Mater. Civ. Eng. 27, 04015027 (2015)

[11] Itasca Consulting Group Inc., PFC3D (Particle Flow Code in Three Dimensions) user's guide (version 6.0), Minneapolis, America (2019) 